\title{
EXTERIOR DIFFERENTIALS OF HIGHER ORDER AND THEIR COVARIANT GENERALIZATION
}

\author{
V. Abramov" and R. Kerner \\ Laboratoire de Gravitation et Cosmologie Relativistes \\ Tour 22, $4^{\text {eme }}$ étage, Boîte 142 \\ Université Pierre et Marie Curie - CNRS URA 769 \\ 4, Place Jussieu, 75005 Paris, France
}

\begin{abstract}
We investigate a particular realization of generalized $q$-differential calculus of exterior forms on a smooth manifold based on the assumption that $d^{N}=0$ while $d^{k} \neq 0$ for $k<N$. It implies the existence of cyclic commutation relations for the differentials of first order and their generalization for the differentials of higher order. Special attention is paid to the cases $N=3$ and $N=4$. A covariant basis of the algebra of such $q$-grade forms is introduced, and the analogues of torsion and curvature of higher order are considered. We also study a $Z_{N}$-graded exterior calculus on a generalized Clifford algebra.
\end{abstract}

\footnotetext{
${ }^{1}$ Permanent address: Institute of Pure Mathematics, University of Tartu, Vanemuise 46, Tartu, Estonia
} 


\section{INTRODUCTION}

An appropriate framework for $d^{N}=0, N \geq 2$ generalization of classical exterior differential calculus (satisfying $d^{2}=0$ ) is provided by the notions of graded $q$-differential algebra and q-differential calculus which have been elaborated in recent papers ( [四], [2], [迎).

Let us remind that a graded $q$-differential algebra is a graded unital algebra over the field $\mathbf{C}$ which is a sum of $N$ algebras with respective grade $k, k \in \mathcal{N}=$ $\{0,1,2, \ldots N-1\}: \mathcal{A}=\oplus_{i \in \mathcal{N}} \mathcal{A}^{i}$, equipped with an endomorphism $d$ of degree one satisfying the $q$-Leibniz rule

$$
d(A B)=d(A) B+q^{a} A d(B), A \in \mathcal{A}^{a}
$$

and such that $d^{N}=0$ whenever $q^{N}=1$. A $q$-differential calculus over an algebra $\mathcal{B}$ is a graded $q$-differential algebra $\mathcal{A}$ such that $\mathcal{B}$ is a subalgebra of $\mathcal{A}^{0}$.

These definitions show the way in which the exterior calculus of differential forms on a smooth $n$-dimensional manifold $M$ should be generalized. The most striking property of this generalized exterior calculus, due to the fact that $d^{N}=0$, is that it contains not only first order differentials $d x^{1}, d x^{2}, \ldots, d x^{n}$ but must also include the higher order differentials $d^{2} x^{1}, d^{2} x^{1}, \ldots, d^{N-1} x^{n}$ as well.

After deriving from the condition $d^{N} f=0$ a set of cyclic commutation relations these differentials must satisfy, it becomes clear that one needs a generalization of the Grassmann algebra in addition to the above definitions in order to produce a self-consistent algebra of generalized differential forms. Such a generalization of Grassmann algebra which displays a representation of the cyclic group $Z_{3}$ by cubic roots of unity has been constructed in (䐗) and then used in a more general form in ([5], [6], [7]) for the construction of the generalized exterior calculus on a smooth manifold. It should be mentioned that differential forms with higher order differentials have been considered in [8], where a formalism of differential forms of higher order on any associative algebra has been developed.

In this paper we continue to study a generalized $q$-exterior calculus on a classical finite-dimensional smooth manifold $M$ paying particular attention to the tensorial behavior of the generalized differential forms under a change of coordinates. The main problem here is that the higher order differentials transform in a non-homogeneous way under a coordinate transformation. In order to circumvent this difficulty we introduce an analogue of linear connection which allows us to replace the ordinary differentials of any order by their covariant generalizations.

The peculiar feature of linear connections we introduce is that due to the higher order differentials its definition includes not only the usual connection coefficients $\Gamma_{j k}^{i}$ but also the additional coefficients $B_{j k}^{i}, C_{j k l}^{i}$ (in the case $N=3$ ) which a priori need not to be iterated by the first order covariant differential. We find the transformation rules of these coefficient functions of a connection which could be called connection coefficients of higher order and we show that $B_{j k}^{i}$ is related to the torsion of a connection. If there is no torsion, then $C_{j k l}^{i}$ can be expressed in terms of the Riemann curvature tensor. 
We also study a particular realization of a $q$-exterior calculus on a generalized Clifford algebra ([9], 10]).

\section{GRADED ALGEBRA OF FIRST AND HIGHER ORDER DIF- FERENTIALS}

Let $M$ be a smooth $n$-dimensional manifold and let $q$ be a $N$-th primitive root of unity $q=e^{2 \pi i / N}, q^{N}=1$. Let $U$ be an open subset of $M$ with local coordinates $x^{1}, x^{2}, \ldots, x^{n}$. Our aim is to construct an analogue of the exterior algebra of differential forms with exterior differential $d$ satisfying the $q$-Leibniz rule

$$
d(\omega \theta)=d \omega \theta+q^{|\omega|} \omega d \theta
$$

where $\omega, \theta$ are complex valued differential forms, $|\omega|$ is the degree of $\omega$, and

$$
d^{N}=0
$$

whereas $d^{k} \neq 0$ for $1<k \leq N-1$. We shall also assume that as in the classical case the exterior differential $d$ is a linear operator and that it increases the degree of a form by one.

Let $U$ be an open subset of $M$ with the local coordinates $x^{1}, x^{2}, \ldots, x^{n}$. A differential form of degree zero is a smooth function on $U$. Thus the set of differential forms of degree zero $\Omega^{0}(U)$ is the subalgebra of a whole algebra which coincides with the algebra of smooth functions on $U$. A differential 1-form on $M$ is an element of a free left module $\Omega^{1}(U)$ over the algebra $\Omega^{0}(U)$ generated by the differentials $d x^{1}, d x^{2}, \ldots, d x^{n}$, and the right module structure on $\Omega^{1}(U)$ is defined by the relations

$$
d x^{i} f(x)=f(x) d x^{i}, \quad f(x) \in \Omega^{0}(U) .
$$

The assumption $d^{k} \neq 0$ for $1<k \leq N-1$ implies that there is no reason to use only the first order differentials $d x^{i}$ in the construction of the algebra of differential forms induced by $d$; one can also add a set of formal higher order differentials, in which case the algebra will be generated by

$$
d x^{1}, \ldots, d x^{n}, \ldots, d^{N-1} x^{1}, d^{N-1} x^{2}, \ldots, d^{N-1} x^{n} .
$$

In order to endow the algebra of differential forms with appropriate $Z_{N}$-grading we shall associate the degree $k$ to each differential $d^{k} x^{i}$. As usual, the grade of a product of differentials is the sum of the degrees of its components modulo $N$. Given any smooth function $f$ and successively applying to it the exterior differential $d$ one obtains the following expressions for the first three steps:

$$
\begin{gathered}
d f=\frac{\partial f}{\partial x^{i}} d x^{i}, \\
d^{2} f=\frac{\partial^{2} f}{\partial x^{i} \partial x^{j}} d x^{(i} d x^{j)}+\frac{\partial f}{\partial x^{i}} d^{2} x^{i},
\end{gathered}
$$




$$
d^{3} f=\frac{\partial^{3} f}{\partial x^{i} \partial x^{j} \partial x^{k}} d x^{(i} d x^{j} d x^{k)}+\frac{\partial^{2} f}{\partial x^{i} \partial x^{j}}\left(d^{2} x^{i}, d x^{j}\right)_{q}+\frac{\partial f}{\partial x^{i}} d^{3} x^{i} .
$$

The relation (3) between left and right structures of the module of 1-forms $\Omega^{1}(U)$ corresponds to classical differential calculus on $U$ (匹11). Because the partial derivatives of a smooth function of classical differential calculus do commute, only the totally symmetric combinations of indices are relevant in these definitions. That is why in the above formulae the parentheses mean the symmetrization with respect to the superscripts they contain, i.e.

$$
\begin{aligned}
d x^{(i} d x^{j)}= & \frac{1}{2 !}\left(d x^{i} d x^{j}+d x^{j} d x^{i}\right), d x^{(i} \\
d x^{j} d x^{k)}= & \frac{1}{3 !}\left(d x^{i} d x^{j} d x^{k}+d x^{j} d x^{k} d x^{i}+d x^{k} d x^{i} d x^{j}\right. \\
& \left.\quad+d x^{k} d x^{j} d x^{i}+d x^{i} d x^{k} d x^{j}+d x^{j} d x^{i} d x^{k}\right) \\
\left(d^{2} x^{i}, d x^{j}\right)_{q}= & d^{2} x^{(i} d x^{j)}+(1+q) d x^{(i} d^{2} x^{j)} .
\end{aligned}
$$

The differentials of higher order of a function $f$ can be expressed by means of a recurrent formula. Let us write the $k$-th differential of a function $f$ in the form

$$
\begin{gathered}
d^{k} f=\frac{\partial^{(k)} f}{\partial x^{i_{1}} \ldots \partial x^{i_{k}}} L_{(k)}^{i_{1} i_{2} \ldots i_{k}}+\frac{\partial^{(k-1)} f}{\partial x^{i_{1}} \ldots \partial x^{i_{k-1}}} L_{(k)}^{i_{1} i_{2} \ldots i_{k-1}} \\
+\ldots+\frac{\partial^{2} f}{\partial x^{i} \partial x^{j}} L_{(k)}^{i j}+\frac{\partial f}{\partial x^{i}} L_{(k)}^{i},
\end{gathered}
$$

where $L_{(k)}^{i_{1} i_{2} \ldots i_{k}}, \ldots, L_{(k)}^{i}$ are homogeneous polynomials of differentials of total degree $k$ symmetric with respect to their superscripts. They can be described by means of the following recurrent formula

$$
L_{(k)}^{i_{1} i_{2} \ldots i_{m}}=d L_{(k-1)}^{i_{1} i_{2} \ldots i_{m}}+\frac{1}{m} \sum_{l=1}^{m} d x^{i_{l}} L_{(k-1)}^{i_{1} \ldots \hat{i}_{l} \ldots i_{m-1}},
$$

for $2 \leq m \leq k-1$, and

$$
L_{(k)}^{i_{1} i_{2} \ldots i_{k}}=d x^{\left(i_{1}\right.} d x^{i_{2}} \ldots d x^{\left.i_{k}\right)}, \quad L_{(k)}^{i}=d^{k} x^{i} .
$$

In order to guarantee that the $N$-nilpotency (2) of the $q$-exterior differential does not depend on the choice of local coordinates, the $N$-th power of the differential $d$ should vanish identically on any smooth function $f$ of a manifold $M$,

$$
d^{N} f=0 .
$$

This leads to the conditions which should be imposed on formal differentials $d x^{1}, d x^{2}, \ldots, \ldots, d^{N-1} x^{1}, d^{N-1} x^{2}, \ldots, d^{N-1} x^{n}$ in order to guarantee (10). In their most general form they are obtained from (7) and can be written as follows:

$$
L_{(k)}^{i_{1} i_{2} \ldots i_{m}}=0, \quad L_{(k)}^{i_{1} i_{2} \ldots i_{k-1}}=0, \quad \ldots, \quad L_{(k)}^{i}=0 .
$$


Let us write these conditions explicitly for the first few values of $N$. If $N=2$ then (11) takes on the form

$$
L_{(2)}^{i j}=d x^{(i} d x^{j)}=0, \quad L_{(2)}^{i}=d^{2} x^{i}=0 .
$$

Obviously these relations generate the classical exterior algebra based on the skew-symmetric Grassmann structure with square nilpotent differential $d^{2}=0$. The first non-trivial generalization of the classical algebra is the case $N=3$ when the conditions (11) take on the form :

$$
\begin{aligned}
L_{(3)}^{i j k} & =d x^{(i} d x^{j} d x^{k)}=0, \\
L_{(3)}^{i j} & =d^{2} x^{(i} d x^{j)}+(1+q) d x^{(i} d^{2} x^{j)}=0, \\
L_{(3)}^{i} & =d^{3} x^{i}=0 .
\end{aligned}
$$

Although this paper concerns mainly with the $N=3$ generalization of differential forms, we also show the constitutive relations (11) for $N=4$ :

$$
\begin{aligned}
L_{(4)}^{i j k l} & =d x^{(i} d x^{j} d x^{k} d x^{l)}=0, \\
L_{(4)}^{i j k} & =d^{2} x^{(i} d x^{j} d x^{k)}+(1+q) d x^{(i} d^{2} x^{j} d x^{k)}+\left(1+q+q^{2}\right) d x^{(i} d x^{j} d^{2} x^{k)}=0 \\
L_{(4)}^{i j} & =d^{3} x^{(i} d x^{j)}+\left(1+q+q^{2}\right) d^{2} x^{(i} d^{2} x^{j)}+\left(1+q+q^{2}\right) d x^{(i} d^{3} x^{j)} \\
L_{(4)}^{i} & =d^{4} x^{i}=0 .
\end{aligned}
$$

The relations (11) represent the minimal set of conditions that should be imposed on the differentials in order to ensure (10). Comparing (111) with (9) we conclude that for any integer $N$ the differentials of first order $d x^{i}$ are $N$-nilpotent :

$$
\left(d x^{i}\right)^{N}=0 .
$$

On the other hand the relations (13) and (14) in special cases of $N=3$ and $N=4$ demonstrate clearly that generally there are no relations implying the nilpotency of any power for the differentials of higher order. Therefore though the algebra generated by the relations (11) is finite-dimensional with respect to the first order differentials because of (15), it remains infinite-dimensional with respect to the entire set of differentials.

Since for $N>2$ the conditions (11) do not represent binary commutation relations, the algebra of differential forms implemented by (11) will be rather hard to work with. One of the ways to circumvent this difficulty is to find relations which on the one hand would be simpler than (11) but on the other hand they would satisfy them. Following this idea we propose to solve the first condition in (13) by assuming that each cyclic permutation of any three differentials of first order is accompanied by the factor $q$ which in this case is a primitive cubic root of unity and satisfies the identity

$$
1+q+q^{2}=0
$$


Thus we assume that each triple of differentials of first order $d x^{i}, d x^{j}, d x^{k}$ is subjected to ternary commutation relations

$$
d x^{i} d x^{j} d x^{k}=q d x^{j} d x^{k} d x^{i} .
$$

These ternary commutation relations can not be made compatible with binary commutation relations of any kind.

Therefore we suppose that all binary products $d x^{i} d x^{j}$ are independent quantities. The second condition in (13) can be easily solved by assuming the following commutation relations:

$$
d x^{i} d^{2} x^{l}=q d^{2} x^{l} d x^{i}
$$

Note that from (17) and (18) it follows that the above ternary and binary commutation relations are coherent in the sense that respect the grading defined earlier, i.e. the quantities $d x^{k} d x^{m}$ and $d^{2} x^{j}$ behave as elements of degree 2 and could be interchanged in the formulae (17) and (18) .

The ternary commutation relations (17) are much stronger than the cubic nilpotence which follows from the first relation of (13). It has been proved in ([5]) that if the generators of an associative algebra obey ternary commutation relations such as (17) then all the expressions containing four generators should vanish. This means that the highest degree monomials which can be made up of the first order differentials have the form $d x^{i} d x^{j} d x^{k}, d x^{i}\left(d x^{j}\right)^{2}$. Thus there are no fourth or higher degree differential forms which can be made up of first differentials. In order to construct an algebra with self-consistent structure we shall extend this fact to the higher order differentials supposing that all differential forms of fourth or higher degree vanish.

Since we have assumed that smooth functions commute with the first order differentials (3), i.e.

$$
x^{k} d x^{m}=d x^{m} x^{k},
$$

then by virtue of the $q$-Leibniz rule the second order differentials do not commute with smooth functions, because differentiating the above equality we obtain

$$
d\left(x^{k} d x^{m}\right)=d x^{k} d x^{m}+x^{k} d^{2} x^{m}=d\left(d x^{m} x^{k}\right)=d^{2} x^{m} x^{k}+q d x^{m} d x^{k},
$$

which leads to the identity

$$
x^{k} d^{2} x^{m}-d^{2} x^{m} x^{k}=q\left(d x^{k} d x^{m}-q^{2} d x^{m} d x^{k}\right)
$$

In what follows, we shall consider only the expressions in which the forms of different degrees are multiplied on the left by smooth functions of the coordinates $x^{k}$, which means that we consider the algebra $\Omega(U)$ as a free finite-dimensional left module over the algebra of smooth functions.

Let us find the number of independent generators $\mathcal{N}$ of this module. We have $n$ first order differentials $d x^{i}$. The number of monomials spanning the module of 2-forms is $n^{2}+n$ because we have $n^{2}$ independent binary products $d x^{i} d x^{j}$ and $n$ second order differentials $d^{2} x^{i}$. The number of monomials spanning the module 
of 3 -forms is $\left(n^{3}-n\right) / 3+n^{2}$ since there are $\left(n^{3}-n\right) / 3$ independent monomials $d x^{i} d x^{j} d x^{k}$ and $n^{2}$ independent monomials $d x^{i} d^{2} x^{j}$. Summing all these numbers one finally obtains the dimension of the module $\Omega(U)$

$$
\mathcal{N}=\frac{n^{3}+6 n^{2}+5 n}{3} .
$$

Although we have described the construction of the algebra $\Omega(U)$ only in the case $N=3$ it can be extended to any integer $N>3$. In this case our algebra is generated by the differentials $d x^{1}, \ldots, d x^{n}, \ldots, d^{N-1} x^{1}, \ldots, d^{N-1} x^{n}$. Let $d^{\alpha_{1}} x^{i_{1}} d^{\alpha_{2}} x^{i_{2}} \ldots d^{\alpha_{r}} x^{i_{r}}$ be a monomial made up of differentials. We shall call the sum $\alpha_{1}+\alpha_{2}+\ldots+\alpha_{r}$ an order of the monomial. For the $N$-th order monomials we shall assume that they are subjected to $r$-cyclic commutation relations

$$
d^{\alpha_{1}} x^{i_{1}} d^{\alpha_{2}} x^{i_{2}} \ldots d^{\alpha_{r-1}} x^{i_{r-1}} d^{\alpha_{r}} x^{i_{r}}=q^{\alpha_{1}} d^{\alpha_{2}} x^{i_{2}} d^{\alpha_{3}} x^{i_{3}} \ldots d^{\alpha_{r}} x^{i_{r}} d^{\alpha_{1}} x^{i_{1}}
$$

where $q$ is a $N$-th primitive root of unity. The relations (17) and (18), which determine the structure of the algebra $\Omega(U)$ in the case of $N=3$, are the special cases of the relations (21). We assume that the monomials of order less than $N$ are independent. For the first order differentials the $r$-cyclic relations (21) take on the form

$$
d x^{i_{1}} d x^{i_{2}} \ldots d x^{i_{N-1}} d x^{i_{N}}=q d x^{i_{2}} d x^{i_{3}} \ldots d x^{i_{N}} d x^{i_{1}} .
$$

Similarly to the case of $N=3$ it can be proved that the above $N$-cyclic relations for first order differentials imply vanishing of all monomials containing more than $N$ first order differentials. Extending this property to the higher order differentials we shall assume that all monomials of order higher than $N$ vanish.

In the next two Sections we show the examples of realization of this exterior calculus. First we discuss the particular properties of a $Z_{3}$-graded one- and twodimensional realizations; then we give an example of $p$ independent differentials acting on a generalized Clifford algebra.

\section{EXAMPLES IN LOW DIMENSIONS}

The aim of this Section is to investigate the structure of the algebra of differential forms introduced above by studying the simplest case of one-dimensional manifold and $N=3$. We shall denote the unique coordinate of this space by $t$.

Differentiating a smooth function $f$ one finds

$$
\begin{aligned}
d f & =f^{\prime} d t \\
d^{2} f & =f^{\prime \prime}(d t)^{2}+f^{\prime} d^{2} t \\
d^{3} f & =f^{\prime \prime \prime}(d t)^{3}+f^{\prime \prime}\left(d^{2} t d t+(1+q) d t d^{2} t\right)+f^{\prime} d^{3} t
\end{aligned}
$$

In this simple case the above definitions yield immediately the relations

$$
(d t)^{3}=0, \quad d t d^{2} t=q d^{2} t d t .
$$


If one does not impose any additional relations, then the algebra of differential forms based on the above commutation relations is infinite-dimensional and it splits into the direct sum of two subspaces

$$
\Omega^{2 m}=\left\{\phi\left(d^{2} t\right)^{m}+\psi(d t)^{2}\left(d^{2} t\right)^{m-1}\right\}, \quad \Omega^{2 m+1}=\left\{\eta d t\left(d^{2} t\right)^{m}\right\} ;
$$

with $\phi, \psi \quad \eta$ smooth functions of $t$.

One has the following rules for calculating the exterior differential

$$
\begin{gathered}
d(d t)=d^{2} t, \quad d\left(d^{2} t\right)=d^{3} t=0, \\
\text { and } d\left[(d t)^{2}\right]=d^{2} t d t+q d t d^{2} t=\left(q+q^{2}\right) d t d^{2} t=-d t d^{2} t .
\end{gathered}
$$

It is interesting that the in their final form the rules for exterior differentiation do not contain the complex parameter $q$.

If $\omega \in \Omega^{2 m}$ and $\omega=\phi\left(d^{2} t\right)^{m}+\psi(d t)^{2}\left(d^{2} t\right)^{m-1}$ then

$$
d \omega=\left(\phi^{\prime}-\psi\right) d t\left(d^{2} t\right)^{m}
$$

which means that $\omega$ is closed if and only if $\phi^{\prime}=\psi$. It is easy to show that any closed differential form of even degree is exact. Indeed, if

$$
\omega=\phi\left(d^{2} t\right)^{m}+\phi^{\prime}(d t)^{2}\left(d^{2} t\right)^{m-1},
$$

then $\omega=d \theta$, where $\theta \in \Omega^{2 m-1}$ and

$$
\theta=\phi d t\left(d^{2} t\right)^{m-1} \text {. }
$$

From this it follows that for any differential form $\theta$ of odd degree one has $d^{2} \theta=0$.

Iterating twice the action of the exterior differential on an even degree differential form $\omega=\phi\left(d^{2} t\right)^{m}+\psi(d t)^{2}\left(d^{2} t\right)^{m-1}$ one obtains the formula

$$
d^{2} \omega=\left(\phi^{\prime \prime}-\psi^{\prime}\right)(d t)^{2}\left(d^{2} t\right)^{m}+\left(\phi^{\prime}-\psi\right)\left(d^{2} t\right)^{m+1},
$$

which shows that $d^{2} \omega=0$ is equivalent to $d \omega=0$. Finally, if $\theta=\eta d t\left(d^{2} t\right)^{m}$ is an odd degree form, then

$$
d \theta=\eta^{\prime}(d t)^{2}\left(d^{2} t\right)^{m}+\eta\left(d^{2} t\right)^{m+1},
$$

and $d \theta=0$ implies $\eta^{\prime}=\eta=0$. Thus any closed form of odd degree is identically null. Now we turn to the transformation laws of differential forms under the change of coordinates. Given a diffeomrphism $t=t(\tau)$ and a differential form of odd degree $\theta=\eta d t\left(d^{2} t\right)^{m}$ one can express it in coordinate $\tau$ as follows

$$
\theta=\left(t^{\prime}\right)^{m+1} \eta d \tau\left(d^{2} \tau\right)^{m},
$$

which gives the transformation law for the coefficient function

$$
\eta=\left(t^{\prime}\right)^{m+1} \eta \text {. }
$$

If $\omega=\phi\left(d^{2} t\right)^{m}+\psi(d t)^{2}\left(d^{2} t\right)^{m-1}$ is a form of even degree then expressing it in terms of coordinate $\tau$ one will obtain

$$
\omega=\left(t^{\prime}\right)^{m} \phi\left(d^{2} \tau\right)^{m}+\left([m]_{q}\left(t^{\prime}\right)^{m-1} t^{\prime \prime} \phi+\left(t^{\prime}\right)^{m+1} \psi\right)(d \tau)^{2}\left(d^{2} \tau\right)^{m-1},
$$

where $[m]_{q}=1+q+q^{2}+\ldots+q^{m-1}$. 
The above formula gives the transformation law for the coefficient functions of a form of even degree:

$$
\phi=\left(t^{\prime}\right)^{m} \phi, \quad \psi=[m]_{q}\left(t^{\prime}\right)^{m-1} t^{\prime \prime} \phi+\left(t^{\prime}\right)^{m+1} \psi .
$$

We end this section by mentioning two facts. The first one is that given any even differential form $\omega=\psi(d t)^{2}\left(d^{2} t\right)^{2 l}$ one can solve the equation $\theta^{2}=\omega$ by letting

$$
\theta=q^{-l} \psi^{\frac{1}{2}} d t\left(d^{2} t\right)^{l} .
$$

We shall denote this solution by $\omega^{\frac{1}{2}}$. The second fact is that given any $2 m+1$ degree form $\theta=\eta d t\left(d^{2} t\right)^{m}$ one can get the closed $2 m$ - degree form by integrating with respect to $d t$, i.e. we define the operator $\mathcal{I}_{a b}: \Omega^{2 m+1} \rightarrow \Omega^{2 m}$ by the formula

$$
\mathcal{I}_{a b}(\theta)=\left(\int_{a}^{b} \eta d t\right)\left(d^{2} t\right)^{m}
$$

where $a<b$ are finite real numbers. These facts gives us a possibility to relate the differential forms we have described with the lenght of a smooth curve on Riemannian manifold. Indeed let $M$ be a Riemannian manifold with metric $g$ and $\alpha:[a, b] \rightarrow M$ be a smooth curve on this manifold which in local coordinates of the manifold $M$ is given by the equations $x^{i}=x^{i}(t), a \leq t \leq b$. Then the first quadratic form $d s^{2}=g_{i j} d x^{i} d x^{j}$ induces by means of the pullback the differential 2 -form

$$
\omega=\alpha^{*}\left(d s^{2}\right)=g_{i j} \dot{x}^{i} \dot{x}^{j}(d t)^{2} .
$$

If we now denote the length of a curve $\alpha$ by $S$ then

$$
S=\mathcal{I}_{a b}\left(\omega^{\frac{1}{2}}\right) .
$$

If we impose the vanishing of all monomials of degree 4 and higher, then on a two-dimensional real manifold with local coordinates $(x, y)$ the left module of $Z_{3}$-graded forms has the dimension 14, as it follows from the general formula (20). One has indeed to take into account the following independent monomials: degree one: $d x, d y$; degree two: $(d x)^{2},(d y)^{2}, d x d y, d y d x, d^{2} x, d^{2} y$, and degree three: $\quad d^{2} x d x, \quad d^{2} x d y, \quad d^{2} y d x, \quad d^{2} y d y, \quad d x d x d y, d x d y d y$

The particularity of the two-dimensional case is that it can be represented in a more elegant way if we introduce complex notation with a single variable $z=x+i y$. Then the 14 independent real expressions above can be expressed as

$$
\begin{gathered}
d z=d x+i d y, \quad d \bar{z}=\overline{d z}=d x-i d y \\
d^{2} z=d^{2} x+i d^{2} y, \quad d^{2} \bar{z}=\overline{d^{2} z}=d^{2} x-i d^{2} y \\
d z d z, \quad d z d \bar{z}, \quad d \bar{z} d z=\overline{d z d \bar{z}}, \quad d \bar{z} d \bar{z}=\overline{d z d z} \\
d^{2} z d z, \quad, d^{2} \bar{z} d z, \quad d^{2} z d \bar{z}=\overline{d^{2} \bar{z} d z}, \quad d^{2} \bar{z} d \bar{z}=\overline{d^{2} z d z} \\
d z d z d z, \quad \text { and } \quad d \bar{z} d \bar{z} d z=\overline{d z d z d \bar{z}}
\end{gathered}
$$


In two real dimensions the expression of a 1 -form $d f$, a 2 -form $d^{2} f$ or of a 2 -form $\omega=d \theta$ with $\theta$ being an arbitrary 1-form are easily computed with the help of general formulae given in the Section 2 .

The situation becomes more interesting if we consider complex holomorphic functions of the variable $z$. In such a case we have only one complex variable and only two independent differentials, $d z$ and $d^{2} z$; there is no more need to introduce their complex conjugates. If we require now that $d^{3} f=0$ for any holomorphic function $f$, then by virtue of

$$
\begin{gathered}
d f=\frac{d f}{d z} d z, \quad d^{2} f=\frac{d^{2} f}{d z^{2}} d z d z+\frac{d f}{d z} d^{2} z, \quad \text { and imposing } \\
d^{3} f=\frac{d^{3} f}{d z^{3}} d z d z d z+\frac{d^{2} f}{d z^{2}}\left[d^{2} z d z+j d z d^{2} z+d z d z\right]+\frac{d f}{d z} d^{3} z=0,
\end{gathered}
$$

we arrive at the conditions on the differentials $d z$ and $d^{2} z$ which are formally the same as the ones verified by the single real variable $t$ :

$$
d^{3} z=0, \quad(d z)^{3}=0, \quad d z d^{2} z=j d^{2} z d z
$$

It is easy to show that the above relations imply that similar ones are verified by the real differentials $d x$ and $d y$ :

$$
d x d^{2} x=j d^{2} x d x, \quad d x d^{2} y=j d^{2} y d x, \quad \text { etc. }
$$

As in the real case, the algebra of degree 1 forms is finite, but there is no reason to cut off the powers of the degree 2 forms $d^{2} z$. We don't see however how the integration introduced for the real one-dimensional case can be generalized to the complex plane.

\section{EXAMPLE OF q-EXTERIOR CALCULUS ON GENERALIZED CLIFFORD ALGEBRAS}

In this Section we shall briefly describe the structure of the generalized Clifford algebra ([9], [10]) and construct a $q$-exterior calculus with $p$ exterior differentials

$d_{1}, d_{2}, \ldots, d_{p}$ each satisfying $d_{k}^{N}=0$. Generalized Clifford algebra is an associative algebra over the complex field whose generators $\Gamma_{1}, \Gamma_{2}, \ldots, \Gamma_{p}$ obey the commutation relations

$$
\Gamma_{i} \Gamma_{j}=q_{i j} \Gamma_{j} \Gamma_{i}, \quad \text { with } \quad \Gamma_{k}^{N}=1,
$$

where

$$
q_{i j}= \begin{cases}1, & i=j \\ q, & i<j \\ q^{-1}, & i>j\end{cases}
$$

It can be proved that the above commutation relations imply the generalized Clifford relation

$$
\left\{\Gamma_{i_{1}}, \Gamma_{i_{2}}, \ldots, \Gamma_{i_{N}}\right\}=N ! \delta_{i_{1} i_{2} \ldots i_{N}}
$$


where the braces $\{, \ldots$,$\} at the left hand side stand for the sum of all$ permutations with respect to the subscripts $i_{1}, i_{2}, \ldots, i_{N}$ which we shall call the $N$-anticommutator and $\delta_{i_{1} i_{2} \ldots i_{N}}$ is the generalized Kronecker symbol which equals 1 when all subscripts are equal and 0 in all other cases. Let us denote the generalized Clifford algebra by $C_{p, N}$. This algebra can be endowed with $Z_{N}$ grading if as usual one associates grade 1 to each generator $\Gamma_{k}$ and defines the grade of any monomial as a sum of the grades of the generators it is composed of modulo $N$. Then the generalized Clifford algebra splits into the direct sum

$$
C_{p, N}=\sum_{i=0}^{N-1} C_{p, N}^{(i)}
$$

where $C_{p, N}^{(i)}$ is a subspace of the elements of grade $i$. The dimension of the vector space underlying the algebra $C_{p, N}$ is $N^{p}$. It can be also proved that the generalized Clifford algebra with $p$ generators is isomoprhic to the grade zero subalgebra of the generalized Clifford algebra with $p+1$ generators, i.e.

$$
C_{p, N} \cong C_{p+1, N}^{(0)}
$$

The generalized Clifford algebras have a matrix representations which can be described as follows. Let us introduce the $n \times n$ matrices

$$
\sigma_{1}=\left(\begin{array}{ccccc}
0 & 1 & 0 & \ldots & 0 \\
0 & 0 & 1 & \ldots & 0 \\
\vdots & \vdots & \ddots & \vdots & \\
0 & 0 & 0 & \ldots & 1 \\
1 & 0 & 0 & \ldots & 0
\end{array}\right), \sigma_{3}=\left(\begin{array}{ccccc}
1 & 0 & 0 & \ldots & 0 \\
0 & q & 0 & \ldots & 0 \\
\vdots & \vdots & \ddots & \vdots & \\
0 & 0 & 0 & \ldots & 0 \\
0 & 0 & 0 & \ldots & q^{N-1}
\end{array}\right)
$$

and $\sigma_{2}=(\sqrt{q}) \sigma_{3} \sigma_{1}$, where $\sqrt{q}$ is needed only in the case when $N$ is an even integer. Let $k=E(p / 2)$. Then the generators of the generalized Clifford algebra $C_{p, N}$ are represented by the $n^{k} \times n^{k}$ matrices

$$
\begin{array}{rcc}
\Gamma_{1}=\sigma_{1} \otimes I^{\otimes(k-1)}, & \Gamma_{2}=\sigma_{2} \otimes I^{\otimes(k-1)}, \\
\vdots & \vdots \\
\Gamma_{2 l-1}=\sigma_{3}^{\otimes(l-1)} \otimes \sigma_{1} \otimes I^{\otimes(k-l-1)}, & \Gamma_{2 l}=\sigma_{3}^{\otimes(l-1)} \otimes \sigma_{2} \otimes I^{\otimes(k-l-1)}, \\
\vdots & \vdots \\
\Gamma_{2 k-1}=\sigma_{3}^{\otimes(k-1)} \otimes \sigma_{1}, \quad \Gamma_{2 k}=\sigma_{3}^{\otimes(k-1)} \otimes \sigma_{2}, \\
\Gamma_{2 k+1}=\sigma_{3}^{\otimes k},
\end{array}
$$

where $I$ is the unit $N \times N$-matrix.

Because the generalized Clifford algebra $C_{p, N}$ possesses a natural $Z_{N}$-grading one can use the $q$-commutator which is defined by the formula

$$
\left[B, B^{\prime}\right]_{q}=B B^{\prime}-q^{b b^{\prime}} B^{\prime} B
$$


where $B, B^{\prime} \in C_{p, N}$ and $b, b^{\prime}$ are the grades of $B, B^{\prime}$. Then $q$-exterior differentials $d_{1}, d_{2}, \ldots, d_{p}$ are defined by the formulae

$$
d_{k} B=\left[\Gamma_{k}, B\right]_{q} \equiv \Gamma_{k} B-q^{b} B \Gamma_{k} .
$$

According to the definition of $Z_{n}$-grading the $q$-exterior differential raises the degree of an element $B$ by 1 , i.e. $d_{k}: C_{p, N}^{(i)} \rightarrow C_{p, N}^{(i+1)}$. It can be proved that each $q$-exterior differential defined in (34) is $N$-nilpotent

$$
d_{k}^{N}=0, \quad \text { for } k=1, \ldots, p .
$$

Indeed if one writes the $l$-th power of the $q$-exterior differential $d_{k}$ in the form

$$
d_{k}^{l} B=\sum_{i=0}^{l} \alpha_{i}^{(l)} \Gamma_{k}^{l-i} B \Gamma_{k}^{i},
$$

then the coefficients $\alpha_{i}^{(l)}$ are found to be

$$
\alpha_{i}^{(l)}=(-1)^{i} q^{\sigma}[l-i+1]_{q}^{(i-1)}, \quad \sigma=\frac{2 a+i-1}{2} i
$$

and

$$
\begin{aligned}
{[l]_{q} } & =1+q+q^{2}+\ldots+q^{l-1} \\
{[l]_{q}^{\prime} } & =1+q[2]_{q}+q^{2}[3]_{q}+\ldots+q^{l-1}[l-1]_{q}, \\
{[l]_{q}^{\prime \prime} } & =1+q[2]_{q}^{\prime}+q^{2}[3]_{q}^{\prime}+\ldots+q^{l-1}[l-1]_{q}^{\prime} \\
{[l]_{q}^{(i)} } & =1+q[2]_{q}^{(i-1)}+q^{2}[3]_{q}^{(i-1)}+\ldots+q^{l-1}[l-1]_{q}^{(i-1)}
\end{aligned}
$$

Thus in order to prove $N$-nilpotence of $q$-exterior differentials suffice it to show that the relation

$$
[N-i+1]_{q}^{(i-1)}=0,
$$

holds for every $i$ from 1 to $N-1$. But this is very easily proved by the mathematical induction with respect to $i$.

It can be also proved that

$$
\left\{d_{i_{1}}, d_{i_{2}}, \ldots, d_{i_{N}}\right\}=0, \quad \text { for } 1 \leq i_{1} \leq i_{2} \leq \ldots \leq i_{N} \leq p .
$$

The above relations follow from (31).

The covariant differentials $D_{1}, D_{2}, \ldots, D_{p}$ can be defined by means of $q$ exterior differentials as follows

$$
D_{k} B=d_{k} B+A_{k} B
$$

where $A_{k}$ is a degree 1 element of the generalized Clifford algebra which we shall call a $k$-component of a connection 1 -form and use the notation $A=$ $\left(A_{1}, A_{2}, \ldots, A_{p}\right)$ combining all components into the connection $A$. 
Now if we apply the operator $\left\{D_{i_{1}}, D_{i_{2}}, \ldots, D_{i_{N}}\right\}$ to an arbitrary element $B$ of the algebra the relations (36) suggest that we get $B$ multiplied by an element of grade zero of the algebra $C_{p, N}$ which we call a $\left(i_{1}, i-2, \ldots, i_{N}\right)$-component of a curvature and denote by $\Omega_{i_{1} i_{2} \ldots i_{N}}$, i.e.

$$
\left\{D_{i_{1}}, D_{i_{2}}, \ldots, D_{i_{N}}\right\}(B)=\Omega_{i_{1} i_{2} \ldots i_{N}} B .
$$

Before giving the explicit expression for $\Omega_{i_{1} i_{2} \ldots i_{N}}$ in terms of connection in a general case we show the expressions for components of curvature in low-dimensional cases of $N=2,3$ and $p=2$. In the case of $N=2, p=2$ the generalized Clifford algebra coincides with the classical Clifford algebra represented by the Pauli matrices

$$
\sigma_{1}=\left(\begin{array}{ll}
0 & 1 \\
1 & 0
\end{array}\right), \quad \sigma_{2}=\left(\begin{array}{cc}
0 & i \\
-i & 0
\end{array}\right), \quad \sigma_{3}=\left(\begin{array}{cc}
1 & 0 \\
0 & -1
\end{array}\right) .
$$

Computing the components of a curvature by means of the formula (37) one obtains

$$
\begin{aligned}
& \Omega_{11}=\left\{\sigma_{1}, A_{1}\right\}+A_{1}^{2}, \\
& \Omega_{12}=\left\{\sigma_{1}, A_{2}\right\}+\left\{\sigma_{2}, A_{1}\right\}+\left\{A_{1}, A_{2}\right\} \\
& \Omega_{22}=\left\{\sigma_{2}, A_{2}\right\}+A_{2}^{2} .
\end{aligned}
$$

If the number of generators $p$ of the algebra remains the same but one takes $N=3$ and denotes by $j$ a cubic root of unity to distinguish it from a generic $N$-th root of unity $q$ then the components of curvature are expressed in terms of the components of connection as follows

$$
\begin{aligned}
& \Omega_{111}=\left\{\eta_{1}, \eta_{1}, A_{1}\right\}+\left\{\eta_{1}, A_{1}, A_{1}\right\}+A_{1}^{3}, \\
& \Omega_{112}=\left\{\eta_{1}, \eta_{1}, A_{2}\right\}+\left\{\eta_{1}, A_{1}, A_{2}\right\}+\left\{A_{1}, \eta_{1}, \eta_{2}\right\}+\left\{A_{1}, A_{1}, A_{2}\right\}, \\
& \Omega_{122}=\left\{\eta_{1}, \eta_{2}, A_{2}\right\}+\left\{\eta_{1}, A_{2}, A_{2}\right\}+\left\{A_{1}, \eta_{2}, \eta_{2}\right\}+\left\{A_{1}, A_{2}, A_{2}\right\}, \\
& \Omega_{222}=\left\{\eta_{2}, \eta_{2}, A_{2}\right\}+\left\{\eta_{2}, A_{2}, A_{2}\right\}++A_{2}^{3},
\end{aligned}
$$

where $\eta_{1}, \eta_{2}$ are the generators of the generalized Clifford algebra $C_{2,3}$ and according to (33) they are represented by the matrices

$$
\eta_{1}=\left(\begin{array}{ccc}
0 & 1 & 0 \\
0 & 0 & 1 \\
1 & 0 & 0
\end{array}\right), \quad \eta_{2}=\left(\begin{array}{ccc}
0 & 1 & 0 \\
0 & 0 & j \\
j^{2} & 0 & 0
\end{array}\right) .
$$

It is worth mentioning that the algebra generated by the above matrices was dubbed by Sylvester the algebra of nonions [12].

The above expressions for the components of a curvature in particular cases $N=2,3$ can be generalized for an arbitrary integers $p, N$ as follows. In order to obtain the expression for the component $\Omega_{i_{1} i_{2} \ldots i_{N}}$ one should take the $N$-th anticommutator of generators $\left\{\Gamma_{i_{1}}, \Gamma_{i_{2}}, \ldots, \Gamma_{i_{N}}\right\}$ and start replacing the generators with the components of a connection with the same subscripts. Let us introduce the following notations. Since there can be equal ones among the integers $1 \leq i_{1} \leq i_{2} \leq \ldots \leq i_{N} \leq p$ and they would give us the same terms we 
pick only different ones denoting them by $1 \leq j_{1} \leq j_{2} \leq \ldots \ldots j_{m} \leq p$ and by $\left|j_{k}\right|$ the number of integers in $\left(i_{1}, i_{2}, \ldots, i_{N}\right)$ equal to $j_{k}$. Then let us denote by $\{A ; \Gamma\}_{j_{k}}$ the anticommutator $\left\{\Gamma_{i_{1}}, \Gamma_{i_{2}}, \ldots, \Gamma_{i_{N}}\right\}$ with $\Gamma_{j_{k}}$ being replaced with $A_{j_{k}}$, by $\{A ; \Gamma\}_{j_{k} j_{l}}$ the same anticommutator with $\Gamma_{j_{k}}, \Gamma_{j_{l}}$ being replaced with $A_{j_{k}}, A_{j_{l}}$ and so on. It should be mentioned that subscripts $j_{k}$ and $j_{l}$ can be equal to each other if $\left|j_{k}\right|>1$. Then the components of the curvature are expressed in terms of connection as follows:

$$
\Omega_{i_{1} \ldots i_{N}}=\{A ; \Gamma\}_{j_{1}}+\ldots+\{A ; \Gamma\}_{j_{m}}+\{A ; \Gamma\}_{j_{1} j_{1}}+\{A ; \Gamma\}_{j_{1} j_{2}}+\ldots+\{A ; \Gamma\}_{j_{1} \ldots j_{m}}
$$

The components of a curvature satisfy the Bianchi identities:

$$
\begin{aligned}
& d_{i_{1}} \Omega_{i_{2} i_{3} \ldots i_{N+1}}+d_{i_{2}} \Omega_{i_{1} i_{3} \ldots i_{N+1}}+\ldots+d_{i_{N+1}} \Omega_{i_{1} i_{2} \ldots i_{N}}= \\
& \quad\left[\Omega_{i_{2} i_{3} \ldots i_{N+1}}, A_{i_{1}}\right]_{q}+\left[\Omega_{i_{1} i_{3} \ldots i_{N+1}}, A_{i_{2}}\right]_{q}+\ldots+\left[\Omega_{i_{1} i_{2} \ldots i_{N}}, A_{i_{N+1}}\right]_{q}
\end{aligned}
$$

\section{COVARIANT BASIS OF $Z_{3}$-GRADED DIFFERENTIALS}

The $q$-exterior calculus on generalized Clifford algebra described in the previous section has a pure algebraic nature and though it is a good model of a generalized exterior calculus with $d^{N}=0$ there even does not arise the question of a change of coordinates. Going back to the algebra $\Omega(U)$ introduced in the section 2 one might ask a question whether this local algebra could be expanded on to the whole manifold $M$. The difficulty here is that the set of generators of the algebra includes the higher order differentials which transform under a change of coordinates in a non-homogeneous way. Our aim in this section is to show that introducing an analogue of a linear connection and replacing the ordinary differentials of all orders with the covariant ones we can overcome the difficulty mentioned above (cf. [6]).

If we suppose that the formal expression $d^{2} f$ does not vanish identically as it is the case in the usual $Z_{2}$-graded exterior calculus of forms, then we must abandon the antisymmetry of the product of 1 -forms in this algebra. The vanishing of the expression (5) could be given an intrinsic sense in any local coordinate system because one supposes that simultaneously $d^{2}=0$, so it applied to any second

differential of a local coordinate, be it $d x^{k}$ or $d^{2} y^{k^{\prime}}$, and parallelly, taking into account the symmetry of partial second derivatives,

$$
\frac{\partial^{2} f}{\partial x^{k} \partial x^{m}}=\frac{\partial^{2} f}{\partial x^{m} \partial x^{k}}
$$

it had to be postulated that the product of 1 -forms must be antisymmetric:

$$
d x^{k} d x^{m}=-d x^{m} d x^{k}
$$

Under a change of local coordinates, $x^{k} \rightarrow y^{m^{\prime}}\left(x^{k}\right)$, and $x^{k}=x^{k}\left(y^{m^{\prime}}\right)$, the basis of 1-forms transformed as a covariant tensor, i.e. $d x^{k}=\frac{\partial x^{k}}{\partial y^{m^{\prime}}} d y^{m^{\prime}}$. However, 
had we abandoned the postulate $d^{2}=0$ and the antisymmetry of the product of 1-forms, the second differentials of the coordinates, which are for the time being purely formal expressions, would not transform as covariant tensors because of the non-homogeneous term:

$$
d^{2} x^{k}=d\left(\frac{\partial x^{k}}{\partial y^{m^{\prime}}} d y^{m^{\prime}}\right)=\frac{\partial^{2} x^{k}}{\partial y^{l^{\prime}} \partial y^{m^{\prime}}} d y^{l^{\prime}} d y^{m^{\prime}}+\frac{\partial x^{k}}{\partial y^{m^{\prime}}} d^{2} y^{m^{\prime}}
$$

Introducing connection coefficients $\Gamma_{l m}^{k}$ we shall define the covariant second differentials $D^{2} x^{k}$ as

$$
D^{2} x^{k}=d^{2} x^{k}+\Gamma_{l m}^{k} d x^{l} d x^{m}
$$

(in order to make our notation homogeneous, from now on we shall also note the first differentials - which are naturally covariant - with capital $D$, i.e. we shall identify $D x^{k}=d x^{k}$.). Note that the above equation can be still interpreted in terms of Grassmann algebra of exterior forms: if we still impose $d^{2}=0$ and the antisymmetry of the exterior product, the covariant 2 -form $D^{2} x^{k}$ is equal to the torsion 2 -form. The vanishing of $D^{2} x^{k}$ is then equivalent to the condition of null torsion, which is valid in all coordinate systems.

Let us suppose now that the differentials $d x^{k}$ and $d^{2} x^{m}$ satisfy the relations imposed by the condition $d^{3}=0$ derived before, i.e., with $q=e^{\frac{2 \pi i}{3}}$ :

$$
d x^{k} d x^{l} d x^{m}=q d x^{l} d x^{m} d x^{k} \quad \text { and } \quad d x^{k} d^{2} x^{m}=q d^{2} x^{m} d x^{k}
$$

It is obvious that these relations remain valid if we replace the ordinary first and second differentials by their covariant counterparts:

$$
D x^{k} D x^{l} D x^{m}=q D x^{l} D x^{m} D x^{k} \quad \text { and } \quad D x^{k} D^{2} x^{m}=q D^{2} x^{m} D x^{k}
$$

which span a covariant basis of the same $Z_{3}$-graded algebra, which has the property of transforming covariantly under the change of a basis.

Now, although $D^{2} x^{k}$ represents a tensorial quantity, its covariant differential $D\left(D^{2} x^{k}\right)=D^{3} x^{k}$ can not be computed by simple iteration, i.e. by applying the same formula as for the covariant differential of $D x^{k}$. As a matter of fact, $D^{3} x^{k}$ has to be a tensorial quantity of third degree, which in covariant basis should contain both $D x^{k} D^{2} x^{m}$ and $D x^{k} D x^{l} D x^{m}$. That is why we should write:

$$
D^{3} x^{k}=D\left(D^{2} x^{k}\right)=d\left(D^{2} x^{k}\right)+B_{l m}^{k} D x^{l} D^{2} x^{m}+C_{l m n}^{k} D x^{l} D x^{m} D x^{n}
$$

with new coefficients of two kinds, whose transformation properties under coordinate change are yet to be derived, and which will assure the tensorial character of $D^{3} x^{k}$. Acting with the operator $d$ on $D^{2} x^{k}$ yields the explicit result:

$$
\begin{aligned}
D^{3} x^{k}=d^{3} x^{k}+ & \partial_{n} \Gamma_{l m}^{k} d x^{n} d x^{l} d x^{m}+\Gamma_{l m}^{k} d^{2} x^{l} d x^{m}+q \Gamma_{l m}^{k} d x^{l} d^{2} x^{m}+ \\
& +B_{l m}^{k} D x^{l} D^{2} x^{m}+C_{l m n}^{k} D x^{l} D x^{m} D x^{n}
\end{aligned}
$$

Now, using the fact that $d^{2} x^{l}=D^{2} x^{l}-\Gamma_{r m}^{l} d x^{r} d x^{m}$, 
we can express $D^{3} x^{k}$ by means of covariant quantities only:

$$
\begin{aligned}
D^{3} x^{k}= & d^{3} x^{k}+\left[B_{l m}^{k}+q^{2} \Gamma_{m l}^{k}+q \Gamma_{l m}^{k}\right] D x^{l} D^{2} x^{m}+ \\
& \quad+\left[C_{l m n}^{k}+\partial_{l} \Gamma_{m n}^{k}-\Gamma_{l m}^{r} \Gamma_{r n}^{k}-q \Gamma_{m n}^{r} \Gamma_{l r}^{k}\right] D x^{l} D x^{m} D x^{m} \\
= & d^{3} x^{k}+\tilde{B}_{l m}^{k} D x^{l} D^{2} x^{l}+\tilde{C}_{l m n}^{k} D x^{l} D x^{m} D x^{n}
\end{aligned}
$$

Now we shall proceed by analogy with the $Z_{2}$-graded case. As we have seen, the condition $d^{3}=0$ implies also the ternary and binary $q$-commutation relations with $q=e^{\frac{2 \pi i}{3}}$. This means that in the final expression we remain with

$$
D^{3} x^{k}=\tilde{B}_{l m}^{k} D x^{l} D^{2} x^{m}+\tilde{C}_{l m n}^{k} D x^{l} D x^{m} D x^{n},
$$

It is obvious that if we want to impose the tensorial behavior on $D^{3} x^{k}$, then both coefficients $\tilde{B}_{l m}^{k}$ and $\tilde{C}_{l m n}^{k}$ must transform as tensors given the manifestly tensorial character of the products of differentials they are contracted with. In contrast, the coefficients $B_{l m}^{k}$ and $C_{l m n}^{k}$ have obviously non-tensorial character, which is compensated by the connection coefficients and their derivatives entering the definitions of $\tilde{B}_{l m}^{k}$ and $\tilde{C}_{l m n}^{k}$. In order to get the transformation rules for the coefficients $B_{l m}^{k}$ and $C_{l m n}^{k}$ we use the observation that the formula (44) implicitly determines how the covariant differential $D$ is acting on the second order differentials. Indeed the left-hand side of (44) can be written in the form

$$
\begin{aligned}
D^{3} x^{k} & =D\left(D^{2} x^{k}\right)=D\left(d^{2} x^{k}+\Gamma_{r s}^{k} D x^{r} D x^{s}\right) \\
& =D\left(d^{2} x^{k}\right)+\frac{\partial \Gamma_{r s}^{k}}{\partial x^{l}} D x^{l} D x^{r} D x^{s}+\left(q \Gamma_{r s}^{k}+q^{2} \Gamma_{s r}^{k}\right) d x^{r} D^{2} x^{s}
\end{aligned}
$$

Before we express $D\left(d^{2} x^{k}\right)$ in terms of the coefficients $B_{l m}^{i}$ and $C_{l m n}^{i}$ let us introduce the following notations. Given whatever quantity $\mathcal{R}_{l m n}$ one can split it into three parts belonging to three representations of the cyclic group $Z_{3}$ :

$$
\mathcal{R}_{l m n}^{k}=\mathcal{R}_{(l m n)}^{k}+\mathcal{R}_{\{l m n\}}^{k}+\mathcal{R}_{(l m n]}^{k},
$$

defined as follows:

$$
\begin{aligned}
\mathcal{R}_{(l m n)}^{k} & =\frac{1}{3}\left(\mathcal{R}_{l m n}^{k}+\mathcal{R}_{n l m}^{k}+\mathcal{R}_{m n l}^{k}\right) \\
\mathcal{R}_{\{l m n\}}^{k} & =\frac{1}{3}\left(\mathcal{R}_{l m n}^{k}+q^{2} \mathcal{R}_{n l m}^{k}+q \mathcal{R}_{m n l}^{k}\right) \\
\mathcal{R}_{[l m n]}^{k} & =\frac{1}{3}\left(\mathcal{R}_{l m n}^{k}+q \mathcal{R}_{n l m}^{k}+q^{2} \mathcal{R}_{m n l}^{k}\right) .
\end{aligned}
$$

Now we can express $D\left(d^{2} x^{k}\right)$ in terms of coefficients $B_{l m}^{i}$ and $C_{l m n}^{i}$ as follows

$$
D\left(d^{2} x^{k}\right)=B_{l m}^{i} D x^{l} D^{2} x^{m}+\left(C_{l m s}^{i}-\Gamma_{r[s}^{i} \Gamma_{l m]}^{r}-\Gamma_{[m s}^{r} \Gamma_{l] r}^{i}\right) D x^{l} D x^{m} D x^{s} .
$$

Differentiating covariantly both sides of (41) one obtains the relation

$$
D\left(d^{2} x^{k}\right)=\frac{\partial x^{k}}{\partial y^{k^{\prime}}} D\left(d^{2} y^{k^{\prime}}\right)-\frac{\partial^{2} x^{k}}{\partial y^{k^{\prime}} \partial y^{l^{\prime}}} \Gamma_{r^{\prime} s^{\prime}}^{l^{\prime}} D x^{k^{\prime}} D x^{r^{\prime}} D x^{s^{\prime}} .
$$


In order to give the transformation rules a more compact form we shall use the following notations

$$
U_{j^{\prime}}^{i}=\frac{\partial x^{i}}{\partial y^{j^{\prime}}}, \quad \partial_{j} U_{k}^{i^{\prime}}=\frac{\partial^{2} y^{i^{\prime}}}{\partial x^{k} \partial x^{j}} .
$$

Then replacing $D\left(d^{2} x^{k}\right)$ and $D\left(d^{2} y^{k^{\prime}}\right)$ in the above formula by their expressions in terms of the coefficients $B_{l m}^{i}$ and $C_{l m s}^{i}$ according to (49) and collecting together similar terms we get the transformation rules

$$
\begin{gathered}
B_{l m}^{i}=B_{l^{\prime} m^{\prime}}^{i^{\prime}} U_{i^{\prime}}^{i} U_{l}^{l^{\prime}} U_{m}^{m^{\prime}} \\
C_{l m s}^{i}=U_{i^{\prime}}^{i} U_{l}^{l^{\prime}} U_{m}^{m^{\prime}} U_{n}^{n^{\prime}} C_{l^{\prime} m^{\prime} s^{\prime}}^{i^{\prime}}+U_{i^{\prime}}^{i} U_{[n}^{n^{\prime}} \partial_{m} U_{l]}^{r^{\prime}} \Gamma_{r^{\prime} n^{\prime}}^{i^{\prime}}+U_{s^{\prime}}^{i} \partial_{r} U_{[n}^{s^{\prime}} U_{l}^{l^{\prime}} U_{m]}^{m^{\prime}} U_{r^{\prime}}^{r} \Gamma_{l^{\prime} m^{\prime}}^{r^{\prime}} \\
+U_{s^{\prime}}^{i} \partial_{r} U_{[n}^{s^{\prime}} \partial_{l} U_{m]}^{t^{\prime}} U_{t^{\prime}}^{r}+U_{i^{\prime}}^{i} U_{[n}^{l^{\prime}} \partial_{l} U_{m]}^{r^{\prime}} \Gamma_{l^{\prime} r^{\prime}}^{i^{\prime}}+U_{s^{\prime}}^{i} \partial_{r} U_{[n}^{s^{\prime}} U_{l}^{m^{\prime}} U_{m]}^{n^{\prime}} U_{r^{\prime}}^{r} \Gamma_{m^{\prime} n^{\prime}}^{r^{\prime}}+U_{s^{\prime}}^{i} U_{t^{\prime}}^{r} \partial_{r} U_{[n}^{s^{\prime}} \partial_{l} U_{m]}^{t^{\prime}}
\end{gathered}
$$

As in the case of torsion in ordinary exterior calculus, the tensorial character is displayed only by the irreducible part of these coefficients displaying the corresponding symmetry.

Here is what we do mean by this. As in the usual case the connection coefficients $\Gamma_{l m}^{k}$ could be split into two parts, the torsion (antisymmetric part) and the symmetric part,

$$
\Gamma_{l m}^{k}=\frac{1}{2}\left[\Gamma_{l m}^{k}+\Gamma_{m l}^{k}\right]+\frac{1}{2}\left[\Gamma_{l m}^{k}-\Gamma_{m l}^{k}\right]=\Gamma_{(l m)}^{k}+S_{l m}^{k}
$$

so the four-index symbols $C_{l m n}^{k}$ as we have mentioned earlier can be split into three parts belonging to three representations of the cyclic group $Z_{3}$ :

$$
C_{l m n}^{k}=C_{(l m n)}^{k}+C_{\{l m n\}}^{k}+C_{(l m n]}^{k} .
$$

Therefore, only the part $\tilde{C}_{[l m n]}^{k}$ has to be taken into account in the final expression:

$$
\begin{gathered}
D^{3} x^{k}=\tilde{B}_{l m}^{k} D x^{l} D^{2} x^{m}+\tilde{C}_{[l m n]}^{k} D x^{l} D x^{m} D x^{n}= \\
=\left(B_{l m}^{k}+q^{2} \Gamma_{m l}^{k}+q \Gamma_{l m}^{k}\right) D x^{l} D^{2} x^{m}+\tilde{C}_{[l m n]}^{k} D x^{l} D x^{m} D x^{n}
\end{gathered}
$$

with

$$
\tilde{C}_{l m n}^{k}=C_{l m n}^{k}+\partial_{l} \Gamma_{m n}^{k}-\Gamma_{l m}^{r} \Gamma_{r n}^{k}-q \Gamma_{m n}^{r} \Gamma_{l r}^{k}
$$

Because the coefficients in both terms on the right-hand side are tensors, we can start to investigate their intrinsic properties. Among these, the condition of reality should be applied to both coefficients separately. Starting with the first coefficient, $\tilde{B}_{l m}^{k}$, and recalling that

$$
q=-\frac{1}{2}+\frac{i \sqrt{3}}{2} \quad \text { and } \quad q^{2}=-\frac{1}{2}-\frac{i \sqrt{3}}{2}
$$

we have explicitly

$$
\tilde{B}_{l m}^{k}=B_{l m}^{k}-\frac{1}{2}\left(\Gamma_{m l}^{k}+\Gamma_{l m}^{k}\right)+\frac{i \sqrt{3}}{2}\left(\Gamma_{l m}^{k}-\Gamma_{m l}^{k}\right)
$$


The imaginary part is the torsion tensor of the connection $\Gamma_{l m}^{k}$, so the reality of the coefficient $\tilde{B}_{l m}^{k}$ is equivalent with the vanishing of the torsion, leaving only the symmetric part of $\Gamma_{l m}^{k}$. So, from now on, we can write

$$
\tilde{B}_{l m}^{k}=B_{l m}^{k}-\Gamma_{l m}^{k}, \quad \text { with } \quad \Gamma_{l m}^{k}=\Gamma_{m l}^{k} .
$$

This means that the $B_{l m}^{k}$ transform as connection coefficients, so that the difference $B_{l m}^{k}-\Gamma_{l m}^{k}$ is a tensor. As a corollary, the vanishing of $D^{3} x^{k}$ implies that $B_{l m}^{k}=\Gamma_{l m}^{k}$ and $\Gamma_{l m}^{k}=\Gamma_{m l}^{k}$.

The symmetry of the connection coefficients makes it possible to identify the tensor appearing in the coefficients $\tilde{C}_{l m n}^{k}$. As a matter of fact, only the part $\tilde{C}_{[l m n]}^{k}$ is relevant here, the other two irreducible parts' contribution vanishing when contracted with the covariant expression $D x^{l} D x^{m} D x^{n}$. The part of $\tilde{C}_{l m n}^{k}$ containing the connection coefficients and their derivatives should be also $Z_{3^{-}}$ anti-symmetrized, yielding

$$
\begin{gathered}
\frac{1}{3}\left(C_{l m n}^{k}+q C_{n l m}^{k}+q^{2} C_{m n l}^{k}+\partial_{l} \Gamma_{m n}^{k}+q \partial_{n} \Gamma_{l m}^{k}+q^{2} \partial_{m}\right) D x^{l} D x^{m} D x^{n} \Gamma_{n l}^{k} \\
-\frac{1}{3}\left(\Gamma_{l m}^{r} \Gamma_{r n}^{k}-q \Gamma_{n l}^{r} \Gamma_{r m}^{k}-q^{2} \Gamma_{m n}^{r} \Gamma_{r l}^{k}-q \Gamma_{m n}^{r}-q^{2} \Gamma_{l m}^{r} \Gamma_{n r}^{k}-\Gamma_{n l}^{r} \Gamma_{r m}^{k}\right) D x^{l} D x^{m} D x^{n}
\end{gathered}
$$

It is not difficult, taking into account the symmetries, to identify the final result in terms of the Riemann tensor: $\tilde{C}_{l m n}^{k} D x^{l} D x^{m} D x^{n}$ is equal to

$$
\left(C_{[l m n]}^{k}+\frac{1}{3}\left[R_{n l m}^{k}+R_{m l n}^{k}\right]+\frac{q}{3}\left[R_{m n l}^{k}+R_{l n m}^{k}\right]+\frac{q^{2}}{3}\left[R_{l m n}^{k}+R_{n m l}^{k}\right]\right) D x^{l} D x^{m} D x^{n}
$$

Taking into account that

$$
C_{[l m n]}^{k}=\frac{1}{3}\left(C_{[l m n]}^{k}+q C_{[n l m]}^{k}+q^{2} C_{[m n l]}^{k}\right)
$$

and assuming that the coefficients $C_{[l m n]}^{k}$ are real, the vanishing of the above expression leads to the equality

$$
C_{[l m n]}^{k}=-\left[R_{n l m}^{k}+R_{m l n}^{k}\right]
$$

The analogy with the usual exterior differential calculus is now obvious. In the usual case the condition $D^{2} x^{k}=0$ implied the vanishing of torsion, $S_{l m}^{k}=$ $\frac{1}{2}\left[\Gamma_{l m}^{k}-\Gamma_{m l}^{k}\right]=0$, whereas now, in our $Z_{3}$-graded case, the similar condition $D^{3} x^{k}=0$ implies not only the vanishing of torsion, but also determines entirely the coefficients $B_{l m}^{k}$ (equal to $\Gamma_{(m l)}^{k}$ ), and partly the coefficients $C_{l m n}^{k}$ namely, their $q$-skew-symmetric part $C_{[l m n]}^{k}$ (equal then to the expression $-\left[R_{n l m}^{k}+R_{m l n}^{k}\right]$ ). By analogy, one can impose similar conditions on the "conjugate" $q^{2}$-skewsymmetric part $C_{\{m n l\}}^{k}$, defining it e.g. as $C_{\{m n l\}}^{k}=C_{[l n m]}^{k}$. However, the totally symmetric part $C_{(l m n)}^{k}$, which is not a tensor, is still undefined, because its contribution cancels automatically when contracted with the 3-form $D x^{l} D x^{m} D x^{n}$. 
The symmetric part of $C_{l m n}^{k}$ together with the coefficients $B_{l m}^{k}$ may be used for the definition of a new kind of parallel transport and generalized geodesic curves. One can define, independently of usual covariant derivative of a vector along a parametrized curve $x^{k}(\lambda)$ determined by given connection coefficients $\Gamma_{l m}^{k}$,

$$
\frac{D Y^{k}}{D \lambda}=\frac{d Y^{k}}{d \lambda}+\Gamma_{l m}^{k} \frac{d x^{l}}{d \lambda} Y^{m}=0,
$$

a second-order covariant derivative which is not an iteration of the first one:

$$
\frac{\mathcal{D}^{2} Y^{k}}{\mathcal{D} \lambda^{2}}=\frac{d^{2} Y^{k}}{d \lambda^{2}}+E_{l m}^{k} \frac{d x^{k}}{d \lambda} \frac{D Y^{m}}{D \lambda}+F_{l m}^{k} \frac{D^{2} x^{l}}{D \lambda^{2}} Y^{m}+G_{l m n}^{k} \frac{d x^{l}}{d \lambda} \frac{d x^{m}}{d \lambda} Y^{n}
$$

where we use a different notation, $\frac{\mathcal{D}}{\mathcal{D} \lambda}$ in order to stress that we don't consider here a simple iteration of the usual covariant differentiation $\frac{D}{D \lambda}$. The coefficients $E_{l m}^{k}$ and $F_{l m}^{k}$ are not identical a priori; all we need to know about the transformation properties of $E_{l m}^{k}, F_{l m}^{k}$ and $G_{l m n}^{k}$ is that the resulting quantity $\frac{\mathcal{D}^{2} Y^{k}}{\mathcal{D} \lambda^{2}}$ transforms as a vector under a coordinate change.

If we replace the vector field $Y^{k}\left(x^{m}(\lambda)\right)$ by the vector $\frac{d x^{k}}{d \lambda}$ tangent to the curve, we obtain a third-order generalization of the geodesic equation:

$$
\frac{\mathcal{D}^{3} x^{k}}{\mathcal{D} \lambda^{3}}=\frac{d^{3} x^{k}}{d \lambda^{3}}+\left[E_{l m}^{k}+F_{m l}^{k}\right] \frac{d x^{l}}{d \lambda} \frac{D^{2} x^{m}}{D \lambda^{2}}+G_{l m n}^{k} \frac{d x^{l}}{d \lambda} \frac{d x^{m}}{d \lambda} \frac{d x^{n}}{d \lambda}=0
$$

Now the $\frac{d x^{k}}{d \lambda}$ etc. are commutative entities, so that $G_{l m n}^{k}=C_{(l m n)}^{k}$. Had we iterated the usual covariant derivative in the above equation, then the coefficients $E_{l m}^{k}+F_{l m}^{k}$ and $C_{(l m n)}^{k}$ would be completely determined from the connection coefficients $\Gamma_{l m}^{k}$ and their derivatives; however, we can introduce more general coefficients having the required transformation properties and independent of $\Gamma_{l m}^{k}$. This is reminiscent of a similar situation one level below, when the Christoffel connection is totally determined by the metric, but a larger class of affine connections exist which are independent of metric.

The generalized geodesic equation of third order (55) defines a larger class of curves that the usual geodesics and may be of interest in probing certain geometrical objects. For example, in the flat Euclidean space the solutions of (55) include not only the straight lines, but also all possible hyperbolae.

The geometric aspects of the new differential calculus are beyond the scope of our present article, and we shall publish them later.

\section{ACKNOWLEDGMENTS}

The authors are grateful to M. Dubois-Violette, O. Suzuki and L. Vainerman for valuable discussions. The first author (V.A.) would like to acknowledge the financial support of the Estonian Science Foundation under the grant No. 2403. 


\section{References}

[1] M. Dubois-Violette, R. Kerner, Universal q-differential calculus and qanalogue of homological algebra, Acta Math. Univ. Comenianae, Vol. LXV, 2 (1996), pp. 175-188.

[2] M. Dubois-Violette, Generalized homologies for $d^{N}=0$ and graded $q$ differential algebras, Contemp. Math., 219, 69-79 (1998).

[3] S. Majid, R. Oeckl, Twisting of quantum differentials and the Planck scale Hopf algebra, Commun. Math. Phys., 205, 617-655 (1999).

[4] R. Kerner, Graduation $Z_{3}$ et la racine cubique de l'équation de Dirac, C.R. Acad. Sci. Paris 312, Sér. II, 191-196 (1991).

[5] R. Kerner, $Z_{3}$-graded exterior differential calculus and gauge theories of higher order, Lett. Math. Phys. 36, 441-454 (1996).

[6] R. Kerner, B. Niemeyer, Covariant q-differential Calculus and its Deformations at $q^{N}=1$, Lett. Math. Phys., 45, 161-176 (1998).

[7] V. Abramov, R. Kerner, B. Le Roy, Hypersymmetry: A $Z_{3}$-graded generalization of supersymmetry, J.Math.Phys. 38 (3), 1650-1669 (1997).

[8] R. Coquereaux, Differentials of Higher Order in Noncommutative Differential Geometry, Lett. Math. Phys., 42, 241-259 (1997).

[9] N. Fleury, M. Raush de Traubenberg and R. Yamaleev, Generalized Clifford Algebras and Hyperspin Manifolds, Int. J. Theor. Phys. 32 (1993), 503.

[10] V. Abramov, Ternary approach to fractional supersymmetry, Proceedings of the XXI International Colloquium on Group Theoretical Methods in Physics, Vol. I, 203 - 207.

[11] A. Borowiec and V. Kharchenko, First order optimum calculi, Adv. in Math., 115, 250 (1995).

[12] J.J. Sylvester, On quaternions, nonions, sedenions, etc., John Hopkins Univ. Circulars 3, 7-9 (1884). 